\title{
Silicone Additives for Paints and Coatings
}

\author{
Alfred Bubat* and Wilfried Scholz
}

\begin{abstract}
Silicone additives are a very widely used type of additive in coatings. Their main feature is the reduction of the surface tension of the liquid coating. Due to this very specific property they are successfully used to overcome surface defects like cratering, poor wetting and others. In addition to this, silicone additives can also enhance the surface slip of a coating, which can be used e.g. to improve the scratch resistance of the surface. The chemistry of the silicone additives and the relationship between chemistry and performance are discussed in this paper.
\end{abstract}

Keywords: Polysiloxanes · Silicones · Substrate wetting · Surface slip · Surface tension

\section{Introduction}

Very often, surface defects occur during and after application of a coating. These defects have a negative influence on both the optical properties of the coating and its protecting quality. Typical defects are:

- Poor substrate wetting

- Crater formation

- Formation of Bénard cells and flooding/floating

- Non-optimal flow (orange peel)

One very significant parameter for all these defects is the surface tension of the materials involved. More specifically, surface tension differences are the reason for these surface defects. The source of such surface tension differences can be within the system itself (solvent evaporation or the crosslinking reaction of the resin) or from external causes (overspray, dust particles, or contamination of the substrate).
To prevent surface tension defects, special additives are used. Many of them influence the surface tension of the paint and/or minimize the surface tension differences. Silicone additives (polysiloxanes) are most widely used in coating formulations.

\section{Usage of Silicone Additives}

Polysiloxanes can - depending on their chemical structure - strongly reduce the surface tension of the liquid paint (Fig. 1). The surface tension of the paint can be stabilized at a relatively low value and surface tension differences cannot develop.
If there is enough incompatibility with the paint system, the polysiloxanes have defoaming properties as well.

Furthermore, silicone additives improve the surface slip of the dried paint film as well as increase the scratch resistance and reduce the blocking tendency.

\subsection{Substrate Wetting}

Substrate wetting depends primarily upon the surface tension of the paint and the critical surface tension of the substrate to be coated. As a general rule, the surface tension of the paint must be lower than or at least at the same level as the surface tension of the substrate. Improper wetting (such as 'crawling' or beadingup of the paint) is to be expected when

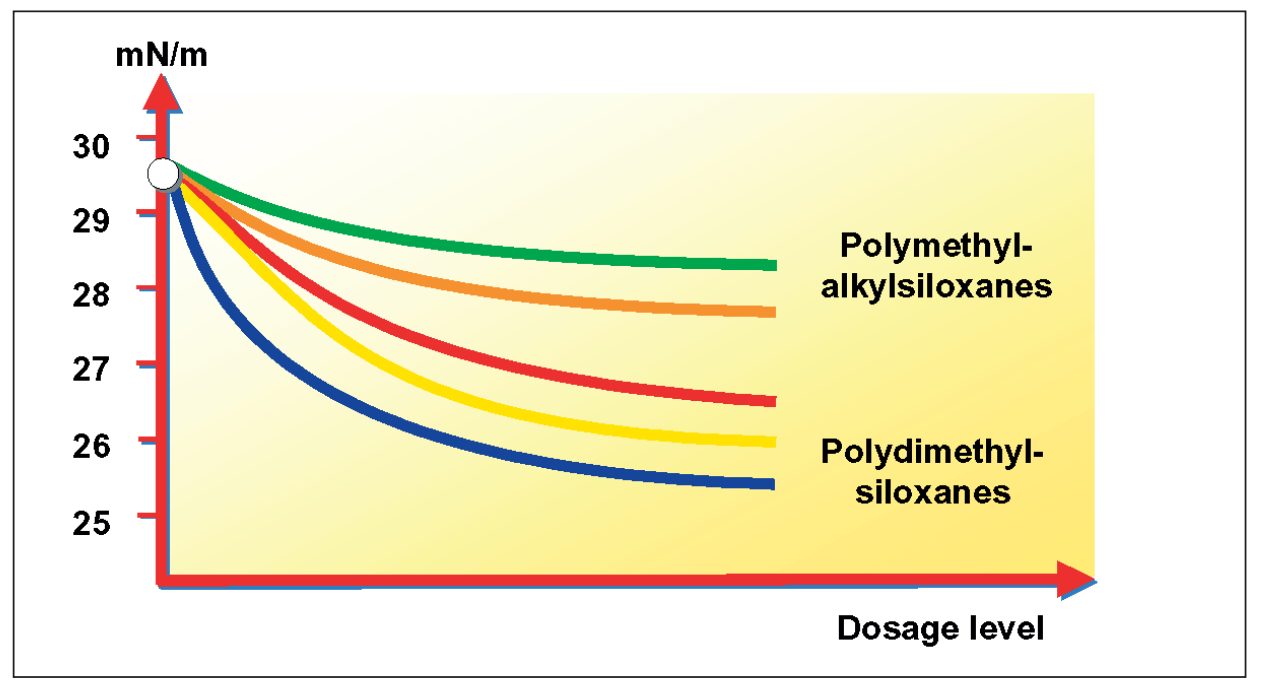

Fig. 1. Surface reduction of a liquid coating by different types of polysiloxanes 
the surface tension of the paint is above the surface tension of the substrate.

Substrates with rather low surface tensions (for example plastics, or substrates with residue of oil or other contamination) are therefore not easily wetted. Water-based paint systems, because of their water content, are obviously higher in surface tension than paint systems with organic solvents, and demonstrate therefore more often problems with substrate wetting.

Silicone additives reduce the surface tension of the paint and accordingly lead to more optimal wetting of the substrate (Fig. 2). For aqueous coatings a special type of silicone additives (silicone surfactants) is available.

\subsection{Anti-cratering}

The causes for crater formation can be very diverse. Craters may be created, for example, by overspray droplets falling into a freshly sprayed paint layer (a layer that is still mobile). Fine spray droplets from the overspray can lead to craters when the surface tension of such droplets is lower than the surface tension of the still mobile paint surface (Fig. 3). Surface tension differences of 1-2 mN/m (dynes/ $\mathrm{cm})$ are sufficient to cause spreading of the droplets, thus leading to craters. In cases where the surface tension of both materials are the same, or if the spray droplets have a higher surface tension, there is no spreading action of the droplets and consequently no crater formation. Small dust particles which fall into the still mobile paint layer can also have the same effect as overspray droplets.

Craters can also originate from uncleaned or contaminated substrates (for example, from fingerprints on panels). When such contaminations display a lower surface tension, they will lead to craters whenever subsequent layers of paint are applied. One can denote the occurrence of such defects as a special case of improper substrate wetting. Through proper utilization of the silicone additive, surface tension is lowered and accordingly the paint becomes visibly less susceptible to disturbances or disruptions from the outside environment (overspray, dust particles, etc.), from the substrate (contamination), or even from the film itself (gelled particles). As a result of the usage of silicone additives, the application of the coating is safer.

\subsection{Bénard Cells, Flooding \& Floating, Surface Flow}

Through the processes of film curing and resin drying, turbulent flow patterns generally arise in coating systems. Such turbulence is constant in nature and transports material from the lower layers of drying film up to the surface of the film. This leads to the formation of macroscopically visible cell structures (Bénard cells, Fig. 4). Differences in density, temperature, and especially surface tension are the driving force behind this cell formation process. Several surface defects like flooding/floating and poor surface flow can be traced back to Bénard cells.

\section{Flooding \& Floating}

In a pigmented paint system, the pigments themselves will participate in the circular flow patterns. In cases where different pigments with different mobilities (different sizes, different densities) are present, such pigments can become widely separated through these flows and disturbances in the film and they are no longer homogeneously distributed. One type of pigment can concentrate in the paint surface and thus change the appear-

Fig. 2. Improvement of substrate wetting by silicone additive due to surface tension reduction
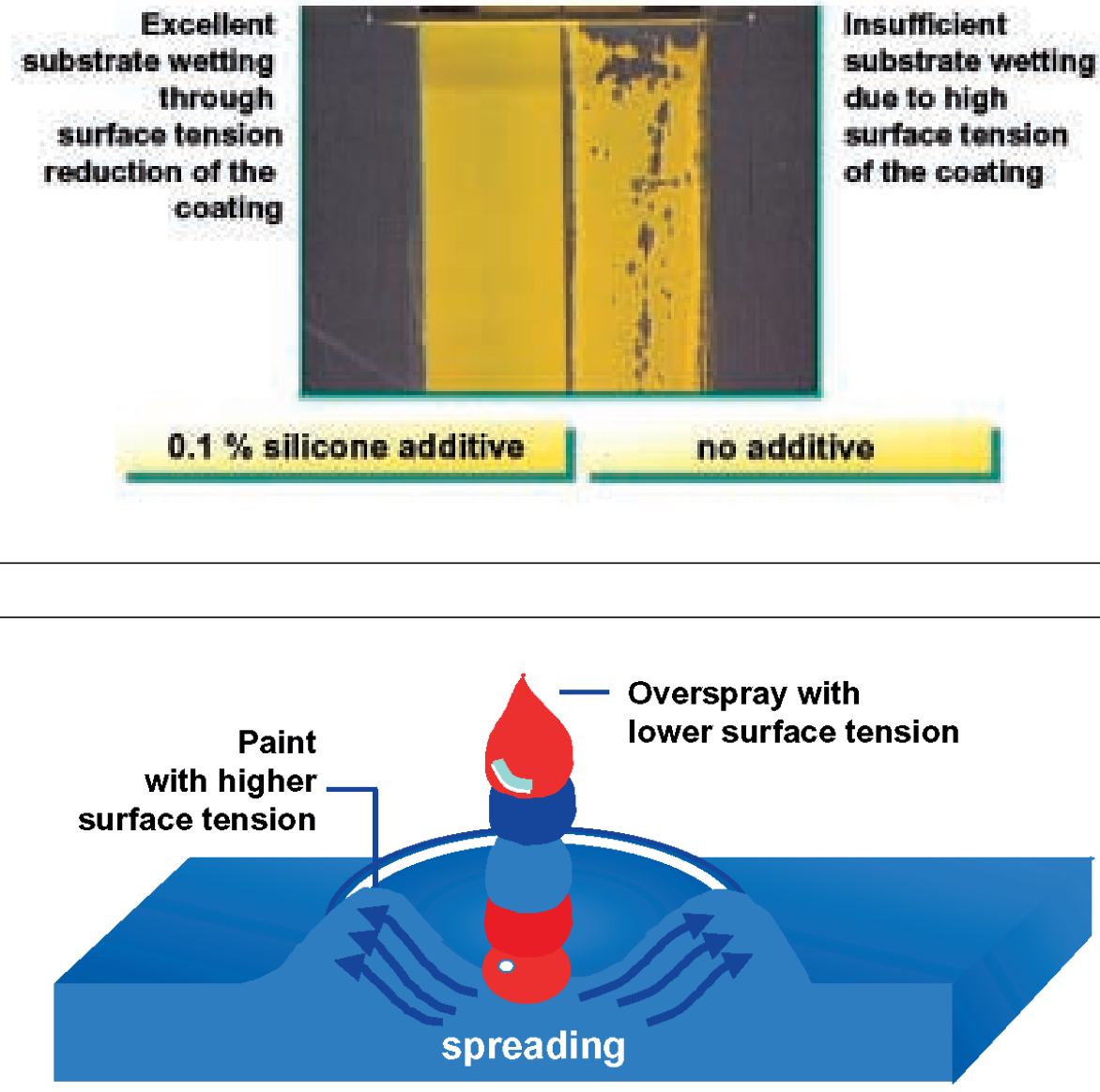

Fig. 3. Formation of a crater in a coating surface due to a overspray droplet (small droplet of a different paint material) that falls in the still liquid coating film 
ance of the coating or even color differences can appear across the surface.

\section{Flow}

The turbulence of the Bénard Cells also leads, in many cases, to paint surfaces that are not uniformly smooth. Sometimes a rather pronounced deformation of the surface texture can occur. The coating shows non-optimal flow - often described as 'orange peel'. Dependent upon the drying conditions and also upon the particular features of the solvent mixture, this effect can occasionally become so strong that the entire top surface of the film is completely disrupted.

When silicone additives are present surface tension differences cannot develop in the surface of the coating film. The circular flow patterns still exist, but in the surface no disturbances become visible and the above-described defects are minimized.

\subsection{Surface Slip}

Silicone additives can improve the slip properties of coating surfaces. Often it is not the mere slip itself, but rather the connection of slip properties with other features of a coating that is of prime interest. Obviously, surfaces with better slip are more scratch resistant, less easily soiled, easier to clean, and more resistant to blocking. Measurement of surface slip can easily be done by monitoring the force necessary to draw a defined weight across the coating surface (Fig. 5, left) or by letting a weight slide down the tilted surface and using the angle at which the weight starts to slide as a measurement for the surface slip (Fig. 5, right).

\subsection{Defoaming}

Foam can, on the one hand, be caused by improper usage of silicone additives. However, on the other hand, the proper usage of silicone additives results in the removal of foam. The crucial factor here is the compatibility of the silicone additive. Highly compatible silicone additives tend to have a higher probability of stabilizing foam. Silicone additives with a controlled incompatibility can be successfully used as defoamers.

\section{Silicone Additives and their Influence on Intercoat Adhesion}

Silicones as a general class of products have an unearned reputation in some corners of the industry for being unrecoatable and for deleteriously affecting intercoat adhesion. However, through the

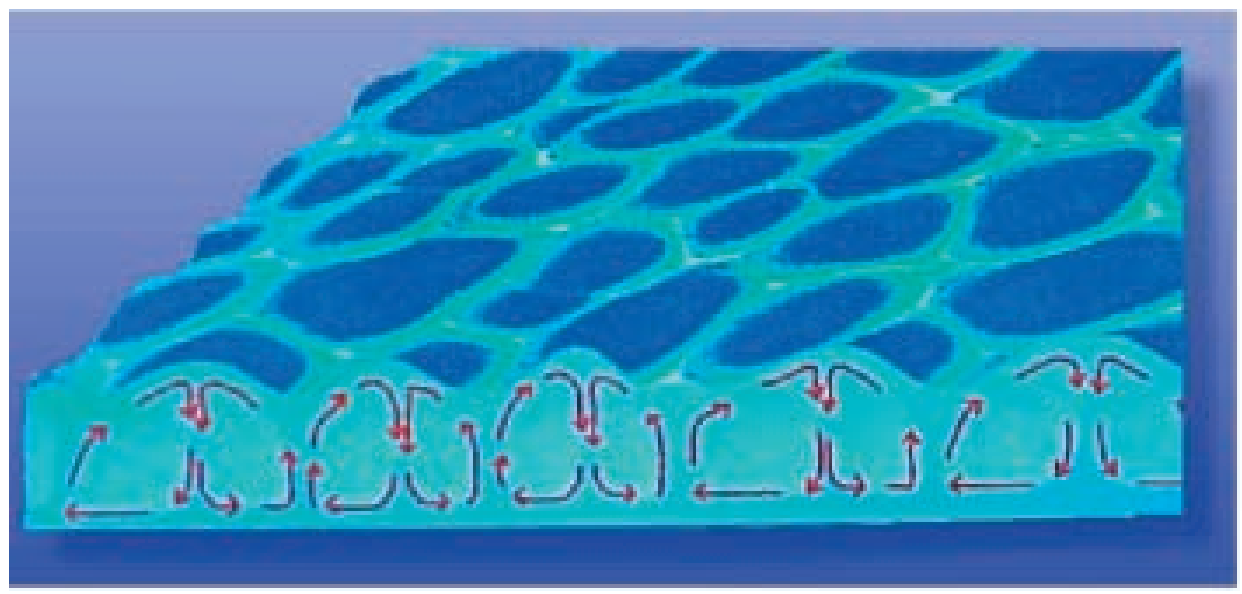

Fig. 4. Formation of Bénard cells in a liquid paint film during the flash-off/drying process

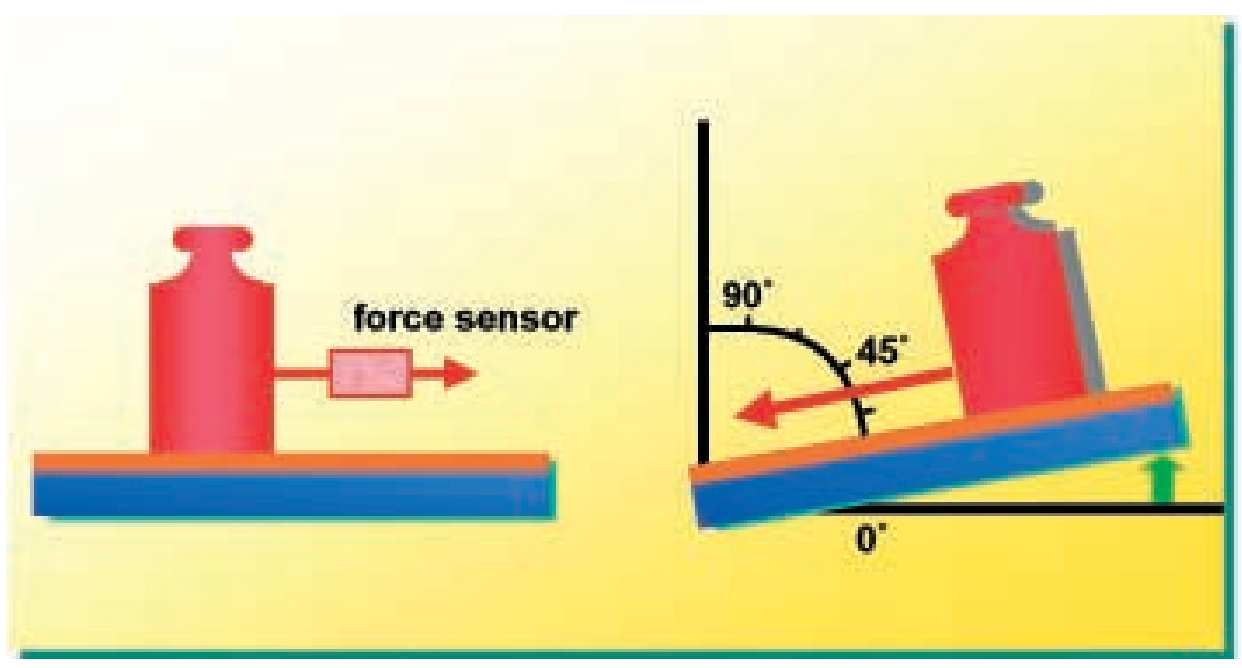

Fig. 5. Measurement of surface slip properties of a coating surface

proper choice and usage of silicone additives, this does not have to be the case. Silicone additives migrate to the paint surface because of their surface activity. Since they, as a rule, have no reactive groups, they will not participate in the drying/curing mechanism of the binder. In other words, this means that silicones remain actively mobile throughout the entire curing process. This is demonstrated, for example, by the fact that one can wipe off or remove the additive from the surface with solvent.

Through the process of overcoating the silicone-containing layer, the silicones do not remain in the surface of the first layer (namely the interfacial layer between the two paint films). In contrast, the silicones migrate because of their mobility and surface activity into the new surface, the second paint layer. Between the two paint films, no silicone remains and, accordingly, the intercoat adhesion is not negatively influenced. In connection with silicones, there are at least two factors which can negatively influence the intercoat adhesion:

- the amount of silicone additive, and

- the curing temperature of the first layer.

For every resin/silicone combination there is an optimal usage level of the silicone. Higher usage levels will not result in additional advantages (wetting, anticrater effect, slip, etc.). In contrast, additional amounts of silicone could bring about undesirable side effects such as loss of intercoat adhesion. Through the overdosage of an additive, some silicone molecules may remain in the interfacial layer between the two paint films and therefore adversely affect adhesion (Fig. 6, upper part). This means that it is very important to adjust the level of silicone so that it is optimal.

In addition to the right dosage, the baking temperature also has an influence on intercoat adhesion. In systems in which the proper baking temperature of the first layer (containing the silicone) is exceeded, the adhesion between the two 
layers will be physically reduced. This is because the polyether chains of the polysiloxanes are decomposed at higher temperatures $\left(140-150^{\circ} \mathrm{C}\right)$ through oxidation. Through this oxidation process, reactive groups are created in the additive so that the silicone additive now becomes an integral part of the coating system and therefore loses its migration ability. By overcoating the system, such decomposition products remain between the two layers and adversely affect adhesion by acting as a release layer (Fig. 6, lower part). Since the thermal instability mentioned above is caused by the presence of polyether chains, it is therefore possible (by more stable groups) to avoid this effect. For example, silicone additives with polyester or aralkyl modifications remain stable at temperatures of up to $220-250{ }^{\circ} \mathrm{C}$ (see sections 4.4.2 and 4.4.3).

\section{Chemistry of Silicone Additives}

Since the beginning of the fifties, silicone additives have played an integral role in coating performance; however, recent chemical innovations have greatly expanded this role, especially in the realm of environmentally friendly systems. A systematic analysis of molecular design is presented with special emphasis upon polyether-modified and polyestermodified polysiloxanes. The chemical determinants of silicone performance are outlined for environmentally friendly coatings, inks, adhesives and related applications. Not only will structure-performance correlations be discussed, but the following topics will be overviewed:

- System compatibility

- Surface tension effects

- Thermostability

- Wetting/leveling

- Slip/mark resistance

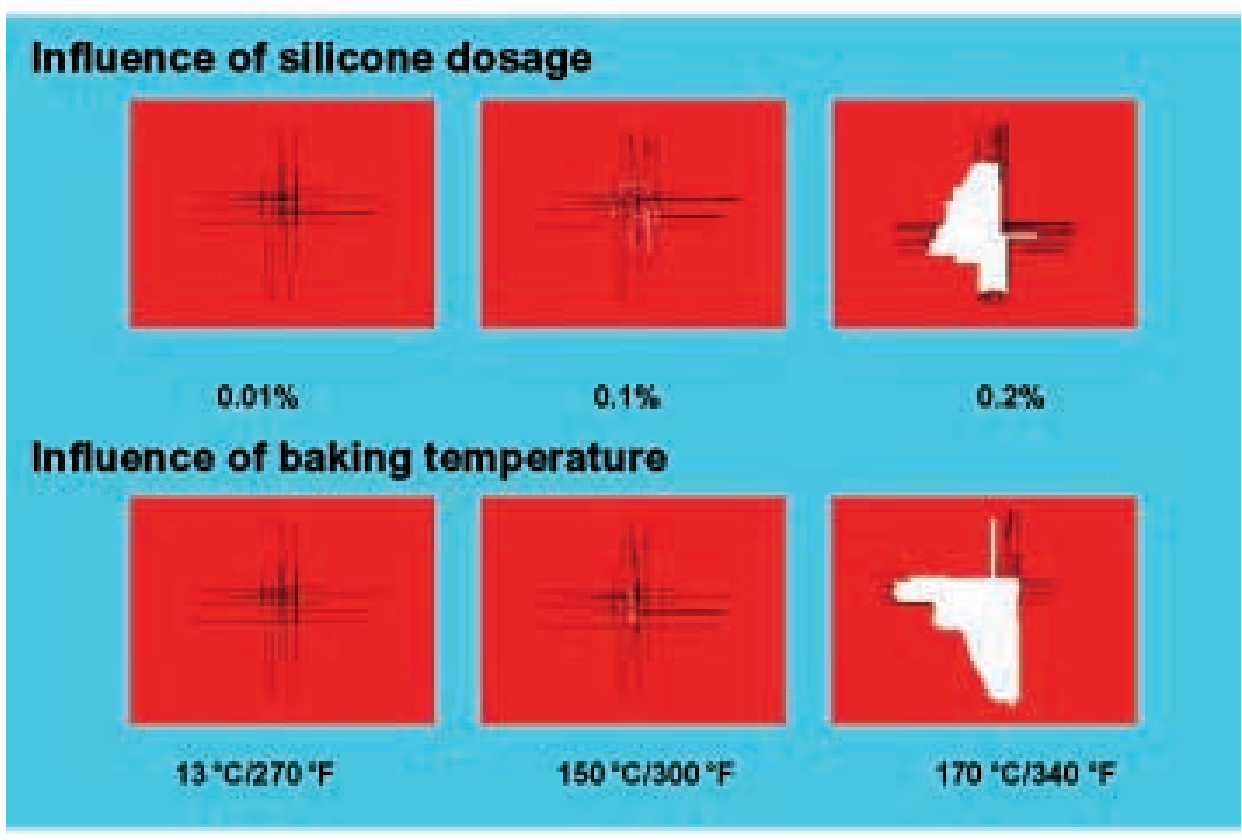

Fig. 6. Influence of silicone additives on intercoat adhesion. The first (white) paint layer contains the silicone additive. After baking it was overcoated with a second (red) layer of paint. After the second baking process intercoat adhesion was tested with the cross cut test

\subsection{Unmodified \\ Polydimethylsiloxanes}

The simplest products are polydimethylsiloxanes, also known as 'silicone oils' in some industry circles. Varying the chain length yields silicones with different properties. With increasing chain length, these materials generally display higher viscosities. Therefore, viscosity is an excellent surrogate measure for the molecular weight of these simple products. Higher molecular weight usually means reduced solubility in coating systems and less compatibility.

In addition to linear products, cyclic products are also possible. The dimethyl groups present in both types of silicones (linear and cyclic) provide the typical silicone effects of surface tension reduction and slip improvement.

Low molecular-weight polydimethylsiloxanes (x < 60, Fig. 7) and cyclic silicones are mainly used to control surface flow and to mask floating of pigments. Their viscosity is between 1 and $50 \mathrm{mPas}$. Such products may be volatile, so their usage levels and conditions are often critically sensitive. After evaporation from the paint film, they can possibly condense and then contaminate other materials, substrates or the after-burning equipment.

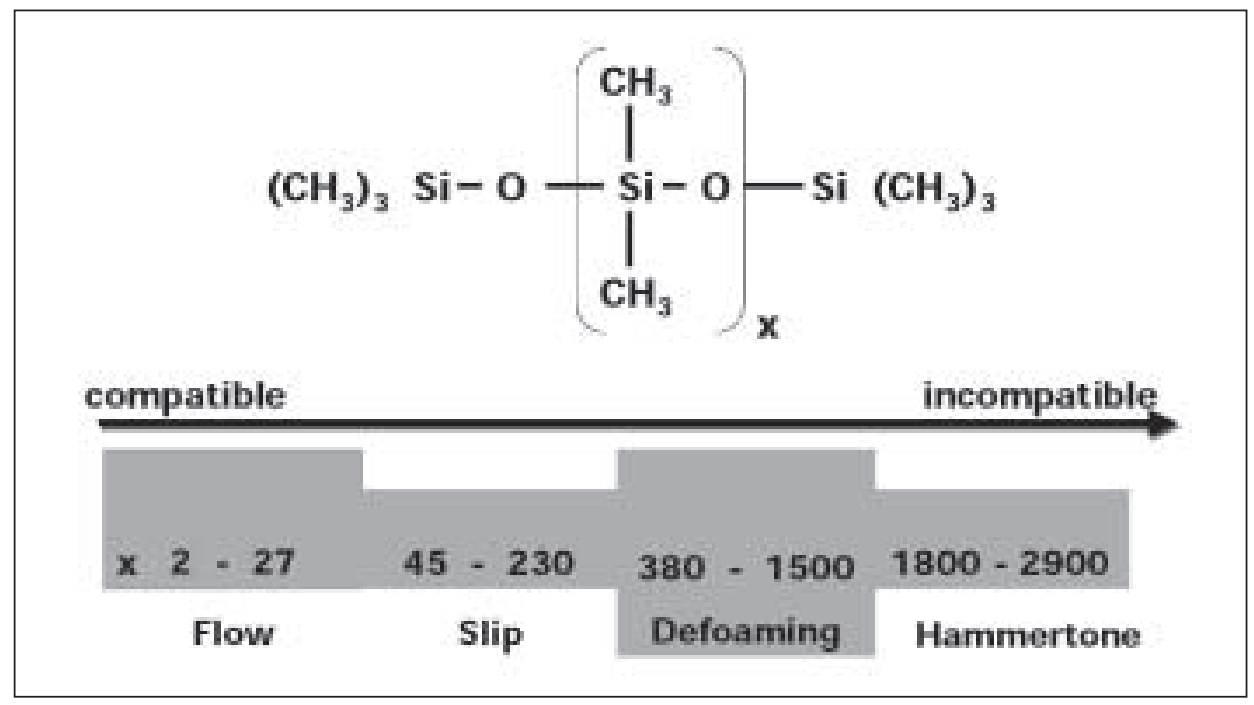


Products with higher molecular weight ( $\mathrm{x}=60-100$, viscosity 50-100 mPas) can additionally be used as slip agents. Polydimethylsiloxanes with ( $\mathrm{x} 1200$, viscosity $\sim 60000 \mathrm{mPas}$ ) often demonstrate just the right balance of compatibility/incompatibility with most common paint resins to be effective as defoamers without creating surface defects. These are classical silicone defoamers.

Finally, those products with very high molecular weight (x >1400, viscosity $>100000 \mathrm{mPas}$ ) are so incompatible and insoluble that they consistently cause craters in nearly all paint systems; therefore, they are relegated to rather limited applications and are generally used only to produce hammertone coatings.

\subsection{Phenylpolysiloxanes}

The pure methylphenylpolysiloxanes (Fig. 8) are of relatively minor importance in the paint industry, even though they are more paint compatible than pure dimethylpolysiloxanes. They influence leveling, but nearly no demonstrable effect on the other properties, such as flooding/floating, slip, and defoaming. If only a few Si atoms in the chain contain phenyl linkages, then products with graduated properties are obtained according to the proportion of dimethyl groups in the molecule. The end result is that slip, leveling, and defoaming can be properly controlled and influenced.

\subsection{Polymethylalkylsiloxanes}

Polysiloxanes with methylalkyl groups (Fig. 9) instead of dimethyl groups still show typical silicone properties but in a less pronounced fashion. They improve surface slip but the influence on surface tension is not as strong as the dimethyl products.

Their degree of activity correlates with the chain length of the alkyl group (Fig. 10).
Fig. 10. Surface tension versus alkyl chain length

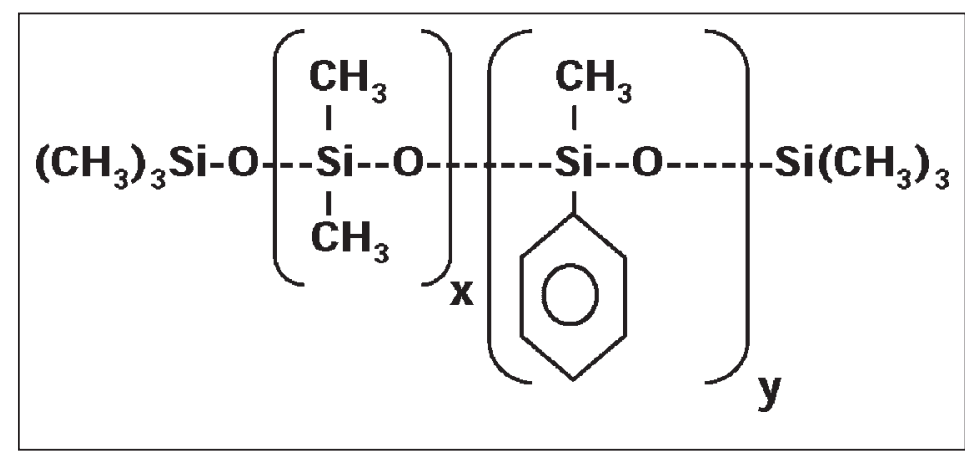

Fig. 8. Phenyl modification

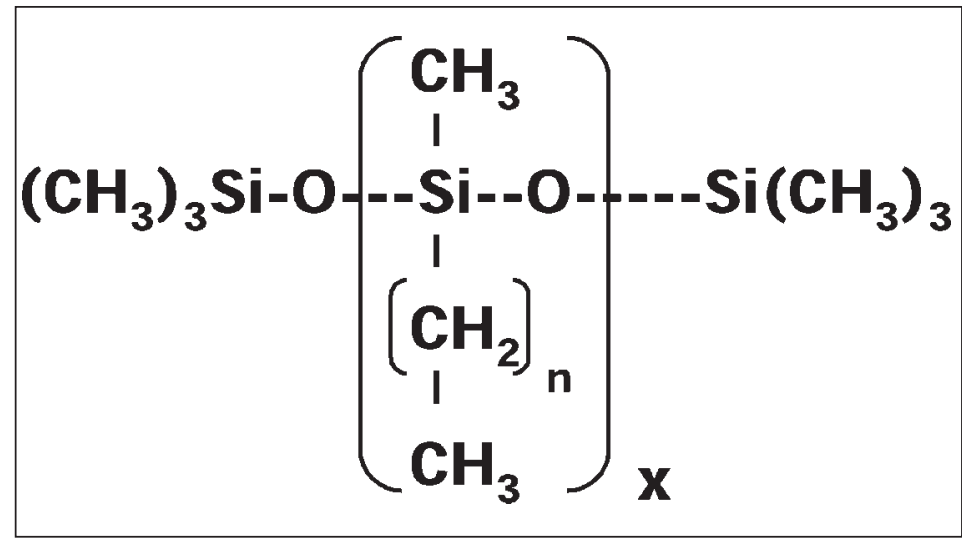

Fig. 9. Methylalkyl modification

Due to the higher surface tension of such products, coatings with these additives are generally easier to recoat in complex multi-layer systems. This type of silicone additive is often also found as the active substance in defoamers.

\subsection{Organically Modified Polysiloxanes}

As explained above, the compatibility of polysiloxanes with coating systems can be controlled, in part, by varying the chain length. However, an infinitely more versatile way to achieve control is by introducing chemical modifications.

\subsubsection{Polyethers}

The most widely used modification is polyether chains (Fig. 11a, 11b). This general chemical structure is so versatile that custom-made silicone additives with very specific properties can be synthesized. Dimethyl groups provide the low surface tension of silicones, so that by

\begin{tabular}{|c|c|}
\hline $\mathbf{n}=$ & $\begin{array}{c}\text { Surface tension } \\
{[\mathrm{mN} / \mathrm{m}]}\end{array}$ \\
\hline 0 & 20.6 \\
\hline 1 & 26.2 \\
\hline 2 & 26.8 \\
\hline 3 & 27.6 \\
\hline 4 & 28.3 \\
\hline 5 & 29.5 \\
\hline 7 & 30.4 \\
\hline 9 & 31.4 \\
\hline 11 & 32.5 \\
\hline 13 & 33.5 \\
\hline
\end{tabular}


changing the ratio of dimethyl groups to organic modifications, the surface tension can be controlled. If, instead of dimethyl groups, methylalkyl groups are present, the resulting products will have considerably higher surface tensions. Thus, they will not be able to reduce the surface tension of the coating as strongly as the dimethyl products.

As mentioned before, the nature of the polyether can be varied; accordingly, this moiety can consist of ethylene oxide (EO) units and/or propylene oxide (PO) units. Polyethylene oxide is strongly hydrophilic (polar), whereas polypropylene oxide is strongly hydrophobic (unpolar); so it is obvious that the resultant ratio (EO/PO) can help to control the overall polarity of the silicone additive. High EO content increases the polarity, so that the silicone becomes water-soluble and therefore more compatible with polar systems; however, the tendency to stabilize foam is also increased. High PO content, on the other hand, reduces watersolubility and increases defoaming properties. In addition to the $\mathrm{EO} / \mathrm{PO}$ ratio, the exact location of the polyether chains in the additive molecule is also critically important. As shown in Fig. 11a and 11b innovative 'end-chain' and 'mid-chain' placement may be achieved in the modern additive structures.

\subsubsection{Polyesters}

It is also possible to use polyester chains rather than polyether groups for modifying the silicone. The resultant polyester modifications (Fig. 12) will also control the compatibility of the silicone product. One very important difference between a polyether-modified and polyester-modified polysiloxane is thermal stability. Polyether-modified products are stable only up to approx. $150^{\circ} \mathrm{C}$.

At higher temperatures, the polyether chain degrades by oxidation (which can be unequivocally proven by IR-spectra). The polyester modification, however, is stable up to $250{ }^{\circ} \mathrm{C}$ before degradation begins. A comparison of various polyester/polyether properties (including heat stability) is shown in the Table.

\subsubsection{Aralkyls}

Aromatic groups can also be used to modify the silicone backbone. The resulting products are, in general, aralkylmodified silicones which display excellent thermo-stability. Aralkyl-modified polymethylalkylsiloxanes (Fig. 13) can combine excellent thermo-stability features with 'higher surface tension effects'- meaning, in the special terminolo- a)

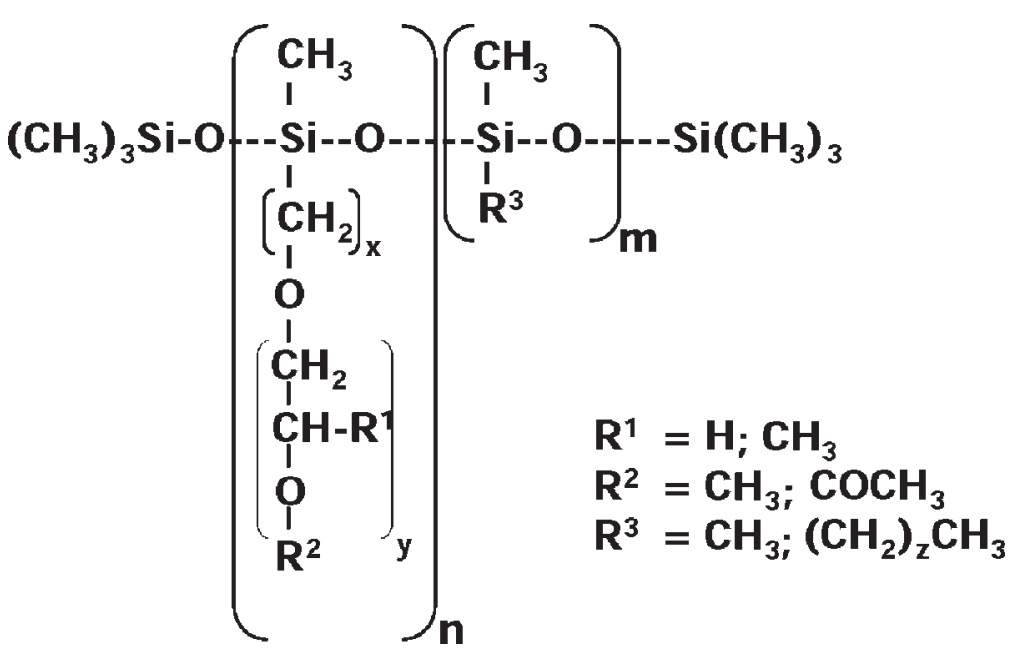

b)

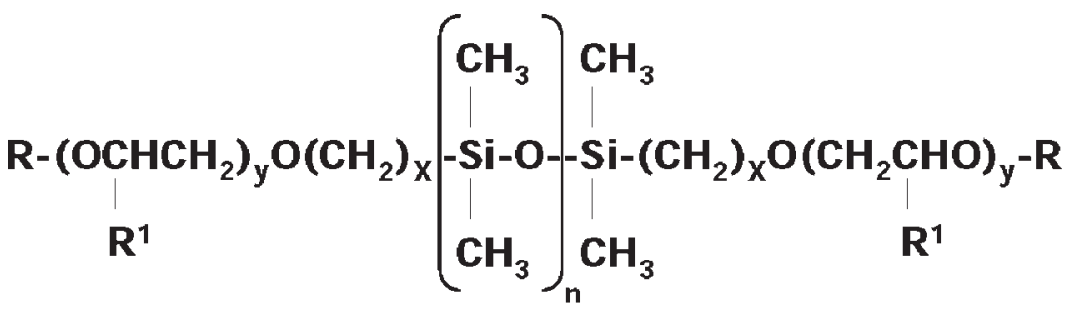

$\mathbf{R}^{1}=\mathbf{H} ; \mathrm{CH}_{3}$

Fig. 11. a) Polyether modification 'mid chain'; b) Polyether modification 'end chain'

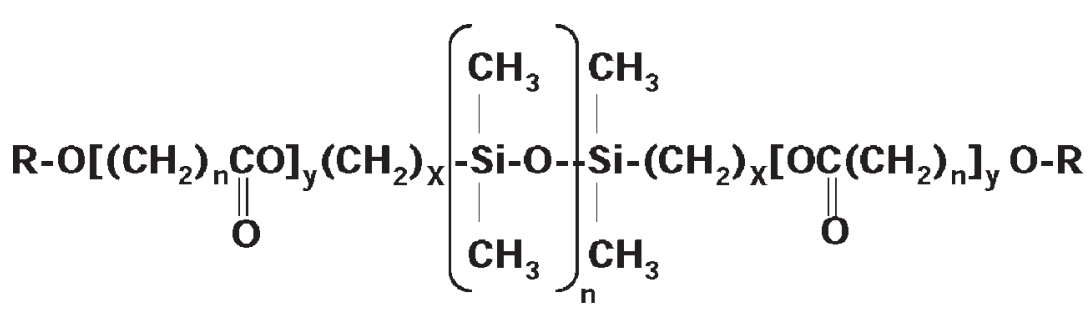

$\mathbf{R}^{1}=\mathbf{H} ; \mathrm{COCH}_{3} ; \mathrm{CH}_{3}$

Fig. 12. Polyester modification Table. Properties of silioxanes

\begin{tabular}{llll} 
Siloxane type & $\begin{array}{l}\text { Polyether } \\
\text { (EO) }\end{array}$ & $\begin{array}{l}\text { Polyester } \\
\text { non-reactive }\end{array}$ & $\begin{array}{l}\text { Pol } \\
\text { r }\end{array}$ \\
\hline Properties & & & 2 \\
Slip properties & 1 & 2 & 2 \\
'Anti-silicone' properties & 1 & 2 & 1 \\
Leveling & 1 & 1 & 5 \\
Recoatability after over-baking & 2 & 1 & 3 \\
Defoaming properties & 4 & 3 & 5 \\
Intercoat adhesion $<\mathbf{1 5 0}^{\circ} \mathbf{C}$ & 1 & 1 & 5 \\
Intercoat adhesion $>\mathbf{1 5 0} \mathbf{~} \mathbf{C}^{\circ}$ & 4 & 1 & 2 \\
Reduction of surface tension & 1 & 2 &
\end{tabular}




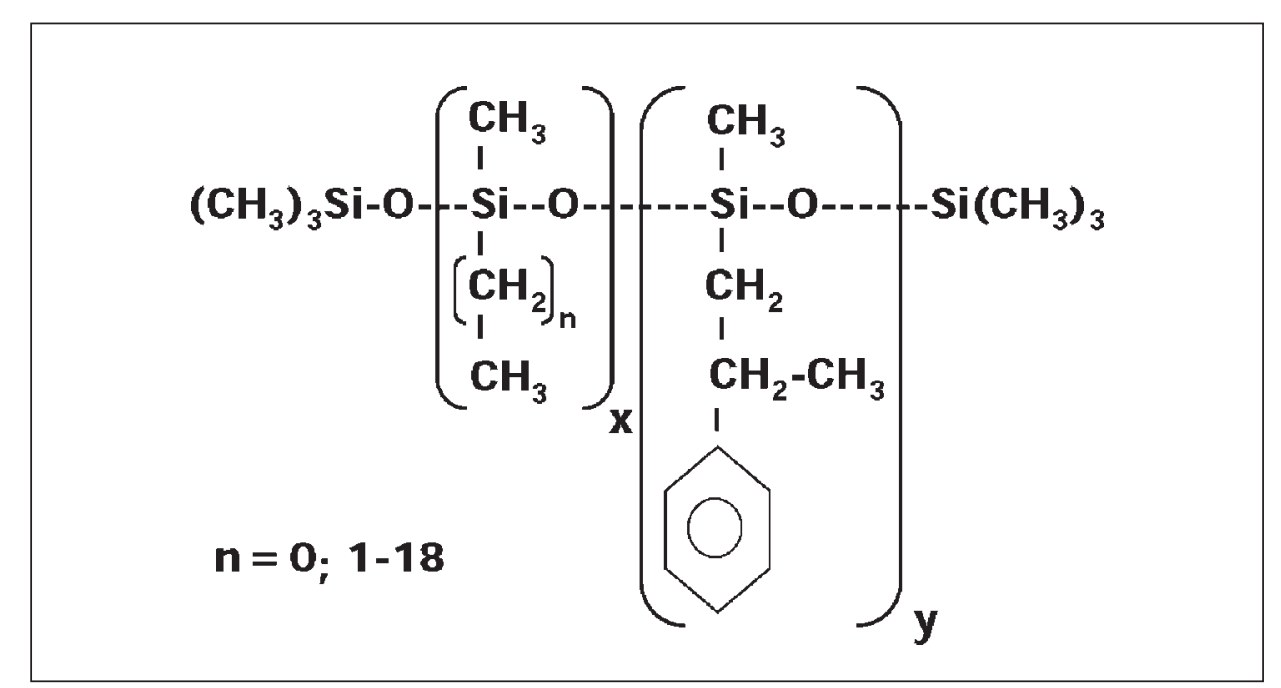

gy of polysiloxane chemists, that surface tension is lowered to a value higher than that achieved with dimethyl moiety. Of course, the higher surface tension effects are due to the presence of methylalkyl groups. If, in addition to the aralkyl and methylalkyl groups, dimethyl groups are also present, the surface tension will drop accordingly.

\subsubsection{Reactive Siloxanes}

Reactive products occupy a special position in the polysiloxane universe; a typical structure is shown in Fig. 14. The general structure of the polysiloxane it- self allows excellent orientation at the surface, while the resin-like polyether or polyester groups contribute to proper compatibility with the binder. The reactive groups are balanced and tailored to the curing mechanism of the paint binders. In summary, the above properties enable excellent cross-linking with paint resin itself. The results are unique surface properties which can generally be achieved only with silicone paints, i.e. permanent, wash-resistant slip, less dirt acceptance, and practically no recoatability.

Received: March 28, 2002
W. Noll, 'Chemie und Technologie der Silicone', Verlag Chemie, 1968.

'Silicone, Chemie und Technologie', symposium monograph, Vulkan-Verlag Essen, 1989.

H.P. Preuss, 'Paint Additives', Noyes Data Corp., Park Ridge, NJ, 1971.

E.W. Orr, 'Performance Enhancement in Coatings', Hanser Publishers, Munich, 1998.
E.W. Orr, 'Silicones in the Coatings Industry: The Influence of Chemical Structure Upon Properties', Lectures available to FSCT Constituent Societies (special monograph series in support of FSCT lectures), 1995-1996.
Fig. 13. Aralkyl modification

Fig.14. General formula of reactive siloxanes 